\title{
ORañarales: espacios simbólicos de cura y peligro Ensayo realizado sobre notas etnográficas con campesinos de comunidades huarpes en el departamento de Lavalle, al NE de la provincia de RMA Mendoza (Argentina)
}

Antropología Social Essay carried out on ethnographic notes with peasants from Huarpe communities in the department of Lavalle, NE in the province of Mendoza (Argentina).

Laura Besio

Grupo de Antropología Social y Ambiental; Instituto Argentino de Nivología, Glaciología y Ciencias Ambientales (IANIGLA); Centro Científico y Tecnológico - CONICET Mendoza. besiolaura@gmail.com

\begin{abstract}
Resumen
El trabajo manifiesta una discusión teórica enmarcada en una investigación mayor aún en proceso sobre saberes y prácticas de cura asociadas a plantas en la región de "Guanacache" (noreste de Mendoza, Arg.). Pobladores que desde la década de 1990 pasaron a personificar un sujeto colectivo huarpe, con reconocimiento de vínculos de continuidad biológica, cultural y social con poblaciones indígenas del pasado. Bajo el supuesto de que las prácticas y saberes trasmitidos que implican el uso de plantas podrían no estar refiriéndose sólo a elementos naturales formalmente ordenados por fuera del sentido social al que dan sentido y contexto, propongo desde una mirada etnográfica construir el carácter histórico y contextual que adquieren los chañarales (bosques nativos de chañar: especie medicinal botánicamente definida como Geoffroea decorticans) en tanto espacios tejidos de sociabilidad en el que transcurren variadas celebraciones rituales: de iniciación de jóvenes en el campo, prosperidad de cosechas futuras, curación de palabra para plantas y animales enfermos, entre otros. Intentaré explicar las formas relacionales en que los chañarales están siendo convocados por la gente local como ámbitos de resistencia espiritual y material para la cura que sincronizan alianzas entre mundos pasados y a la vez presentes.
\end{abstract}

Palabras clave: Guanacache; bosque de chañar; ambiente; plantas curativas; magia y ritual.

\begin{abstract}
Abtract
The work manifests a first theoretical discussion framed in further research still in the process of healing knowledge and practices associated with plants in the region of "Guanacache" (northeast of Mendoza, Arg.), settlers from the 1990s came to personify a collective subject huarpe, with appreciation links biological, cultural and social continuity with the past indigenous populations. Under the assumption that the practices and transmitted knowledge involving the use of plants could not be referring only to natural elements formally ordered outside the social sense to give meaning and context, I propose from an ethnographic look to build the historical and contextual character acquire chañarales (native forests consist mainly of chañar: medicinal species botanically defined as Geoffroea decorticans) while tissue spaces of sociability in various ritual celebrations that take place: initiation of young people in the field, prosperity of future harvests, healing word to disease of plants and animals, among others. My focus of study are healing plants and by employing ontological theories hybrid society nature that try to explain the relational ways that chañarales would be called by the local people as areas of spiritual strength and material for the cure that synchronize alliances between past worlds and simultaneously present.
\end{abstract}

Keywords: Guanacache; chañar forest; environment; healing plants; magic and ritual.

"...Para la tos, té de chañar brea, arrope de chañar criollo, entrecáscara de chilca, con corazón de coronta. Para la embriaguez, sesos de lechuzo, preparado en té en pequeña cantidad"1

\footnotetext{
${ }^{1}$ Narrado por Cecilia de Ortiz, de 79 años. Inscripto en la Encuesta Nacional Folclórica (ENF), formalizada por la Facultad de Filosofía
}

Uno aquí, otro más allá y en las amplias cercanías emergen terceros y cuartos. Chañarales que denotan la forma del

y Letras de la Universidad de Buenos Aires, Instituto de Literatura Argentina bajo la dirección de Ricardo Rojas en el año 1921. La edición de la ENF se completó en Buenos Aires, Imprenta de la Universidad (1937), donada por el Consejo Nacional de Educación al Instituto Nacional de Antropología y Pensamiento Latinoamericano (IAPL). 
campo cuando estamos sobre tierras propias y propensas a ser lagunas, a ser canales, a ser agua de crecida. De día, nuestra mirada busca taparse y escabullirse del inabarcable horizonte en el anonimato de estos bosques que hacen de refugio, de suspiro y respiro, y de ceremonia para velar por prósperas cosechas y para la cura de enfermedades de los huertos. De noche, espacio asociado a un habitáculo, al sonido de guitarras, a brujas, a ceremonias de iniciación "... porque la corteza de chañar cuando hay viento suena de formas que estremecen". Transitado de día o de noche, el contexto adecuado para ser contado es precisamente bajo su dosel, inmerso en su presencia. No afuera, no lejos. No ausente.

Chañaral es una forma que tiene la gente en el campo de llamar el espacio de bosque que emerge en grandes proporciones a lo largo y ancho de la travesía de Guanacache (noreste de la provincia de Mendoza, Argentina); es un espacio enmarcado por frescos descascarados troncos erguidos con recta simetría, solitarios aunque casi siempre aunados bajo tierra por una única raíz madre; otras veces acompañados por la envoltura suave de pastos que irrumpen entre las espinosas ramas de pinchagua y de lámaro².

Un chañaral en la región de Alto con Retamo, en la zona de las Lagunas del Rosario (departamento de Lavalle, noreste de la provincia de Mendoza), es protagonista del devenir de tierras y aguas, contenciones y desplazamientos comunitarios que lo conformaron durante años en un espacio de potrero, hoy convertido en una cerrazón que se muestra todavía contenida dentro de una construcción de enramada, junto a los vestigios del canal derivador construido antiguamente para regar el potrero y así aprovechar el agua de crecida que de otro modo se hubiera "enlagunado y perdido". Una forma de manejo del riego en el monte que consistía en encausar el agua de crecida del río mediante canales construidos entre distintas personas de la comunidad; agua que luego discurría lentamente por el terreno de aquel potrero gracias a un manejo de la pendiente en manos de quienes hacían posible un continuado fluir del deslizamiento del agua que ingresaba al potrero; cuyo control era evidenciado por la altura del agua reflejada en palitos clavados en la tierra que iban dirigiendo posteriores necesidades de nivelación del terreno a favor de un movimiento laminar lento pero que a su vez prometiera evitar estancamientos de agua y así regar la totalidad del monte viviendo allí dentro. De este modo aquel potrero funcionaba como una isla que proveía de vegetación a los animales en épocas en las que la comida en el monte escaseaba o en momentos de parición en invierno para alimentar a las cabras parturientas y/o a sus crías.

\footnotetext{
2 Pinchagua es la denominación local para algunas especies vegetales del género Lycium spp., con mayor frecuencia en mis notas de campo la gente refiere como tal a la especie $L$. tenuispinosum; mientras que la referencia de lámaro se corresponde con la denominación botánica de la especie Prosopis alpataco.
}

La vida en el monte en las formas que toma el terreno que aseguran el buen escurrimiento, en los canales que llevan eventualmente agua de crecida de río, en las altas enramadas siempre renovadas de poda y armazón, y en el espacio intransitable creciendo entre chañares que no hacen más que recordar lo inútil que resulta anclar la mirada más allá del imprevisto movimiento que encuentra paso a paso el cuerpo en su intento por alojarse allí dentro, aunque más no sea por un instante de relato. Eso es el ambiente, una reunión improvisada con los procesos formativos de vida "para adelante"; formas que cambian y en su andar van trazando canales de vida: lo que es tierra de lagunas, deviene de la mano de personas que construyen embanques que propician el enriquecimiento de tierra en alimento para el ganado en la escasez del invierno, y es a su vez fuga de vida futura que se transforma en el pasar de los años en un bosque de chañar en el que hoy son relatadas ceremonias pasadas con aire de presente.

En tal caso, el bosque de chañar o chañaral es: ¿un objeto ecosistémico dominado por árboles de la especie nativa Geoffroea decorticans?; o iun objeto sujeto a aprovechamientos forestales, culinarios y medicinales por la gente que vive en sus cercanías?; o ¿un sitio histórico que conserva los vestigios de lo que antiguamente fue un potrero y con él la permanencia de antiguas prácticas indígenas?; ¿o es sus sombras que conviven con el relato de ceremonias pasadas teñidas de suspicaz actualidad? Según intentaré desarrollar en el presente trabajo, el chañaral es a la vez todas las respuestas; es una extensión de vida que toma forma según reclame la intención de los curiosos, sea éste un reclamo proclive de sociedad, historia, economía, cultura o de naturaleza. Es en sí mismo un lugar donde varios aconteceres se entrelazan y devienen en seres impenetrables que contienen exclusividad en el relato de ceremonias realizadas bajo sus sombras.

Muchos son los intelectuales que ponen en relieve la falta de cuestionamiento en el empleo de herramientas epistemológicas, clasificatorias y analíticas propias de la racionalidad occidental en el momento de abordar la sensibilidad cotidiana con la que diversos grupos sociales se relacionan con su ambiente (Descola 2001, 2012; Descola y Palsson 2001; Ingold 2001, 2004, 2006, 2012b; Marconetto 2008; Latour 1991; Palsson 2001; Rival 2004; Surallés 2004). En los siguientes párrafos me propongo situarme en consenso con los autores que se distancian teórica y metodológicamente de la dualidad naturaleza/sociedad y poner el foco en el espacio denominado chañaral, como una entidad vegetal objeto de interpretaciones según discursos locales de tradición de principios del siglo $X X$, y según enunciaciones de la gente local percibidas actualmente en el propio trabajo de campo etnográfico. Las reflexiones y elementos de etnografía expuestos en este texto intentan tomar en cuenta al chañaral como un campo englobante de relaciones en el que la vida transcurrirría sin reduccionismos. 


\section{La incidencia del suelo}

Desde una mirada ontológica sobre la incidencia del suelo en la realidad estudiada, la antropología desafía los cuerpos de supuestos y prácticas que previamente la tradición occidental ha desatendido por tratarse de conocimientos que no se ajustan a las reglas canónicas de la modernidad, y por tanto por ella no han sido sistematizados ni universalizados. La ontología indaga acerca de la naturaleza de la realidad; y tiene precedencia sobre el campo de la epistemología porque cuestiona precisamente cómo el conocer algo presupone lo que eso es; " es la excavación de la epistemología, un catálogo de los pasos que se dan y los supuestos utilizados para llegar a una visión particular de las cosas" (Weiner en Wright 2008:33).

Rodolfo Kusch, en su obra sobre pensamiento indígena y popular americano, argumenta sobre una ontología y una historia propia de la región; donde el vínculo de las personas con su entorno es una serie de aconteceres, que a diferencia de la idea originada por el pensamiento occidental, no implica en lo absoluto un proceso de objetivación. En este sentido, el autor explica cómo "lo dado (es) todo lo que está, desde lo visible hasta el mero darse de prójimos, dioses y demonios, y es, por eso mismo, lo indeterminado, pero que funda desde lo impensable, y donde lo que es no pasa de ser un mero episodio" (Kusch en Wright 2008 : 37). De este modo, las sociedades americanas viven el estar-siendo, y estar implica no objetos sino eventos que conforman (aunque más no sea fugazmente) el campo complejo de relaciones englobante entre humanos y otras entidades significativas (Kusch 2012).

En palabras de Descola y Palsson (2001), el abordaje de un análisis de las relaciones de los grupos humanos con su medio ambiente debería resaltar la relacionalidad antes que las disyunciones definidas a priori, y de hecho ampliar el foco hacia los contextos sociales del saber y de las prácticas ambientales que están por fuera del campo ontológico descripto como Naturalismo (Descola 2001, 2012) que encuentra "natural" sus propios presupuestos, y construye saberes sobre la creencia de que la naturaleza efectivamente existe y de que ciertas cosas deben su existencia y su desarrollo a un principio ajeno tanto a la suerte como a los efectos de la voluntad humana. En este sentido, la construcción de una naturaleza y una sociedad como campos autónomos puros es el punto esencial de la constitución moderna que vuelve invisible, impensado e irrepresentable el espacio de transición que reúne elementos híbridos, es decir, aquellos elementos que son tanto naturales como sociales (Latour 1991) y que representan en sí mismos las formas propias que tiene la gente de interpretar su mundo, en términos de ontología y no de una "visión del mundo".

Hviding (2001) formula una crítica a las etnociencias convencionales por su incapacidad de incorporar "etnoepistemologías" alternativas y su correlativa tendencia a reificar ciertos dominios de conocimiento indígena para hacerlos compatibles con la ciencia occidental. Estas tendencias, dice “...impiden cualquier comprensión seria del papel que desempeñan ciertas creencias y prácticas, como la magia y el ritual, en la relación diaria de las personas con su ambiente" (p. 210).

En este sentido, Escolar (2012) en un estudio sobre salamancas ${ }^{3}$ argumenta cómo la magia instituye un espacio y un tiempo escurridizo de producción de nociones de lo real por constitución y destitución de identidades y sujetos políticos indígenas, convirtiéndolo en un modo de resistencia de las fuentes de saber dentro de contextos en que fenómenos mágicos son epistemológica y ontológicamente inaprensibles. En palabras del autor,

"las salamancas son a su vez museos, archivos
y laboratorios: tienen sus expertos o brujos, sus
eventuales soberanos que buscan en ellos las
fuentes de su conocimiento, orientación en la
incertidumbre, a la vez que el poder y la posibilidad
de realizar o destituir lo real del presente y del
pasado." (p.18).

Eventos que constituyen el estar-siendo, devenires en magia como espacios repletos y repletados de sentido para la vida de la gente en su relación con el ambiente; relaciones desplegadas en la magia que son a su vez parte de la comprensión ecológica exhaustiva de cómo interactúan las personas reales con los ecosistemas y de la sensibilidad y habilidad con que lo hacen. En esta línea conceptual, el abordaje desde un principio relacional del ser nos ayuda a pensar cómo y desde qué lugar ontológico humanos y no humanos existen inmersos en un medio ambiente en esencia relacional, donde la relevancia está dada en las intensidades que muestran esas relaciones en su caminar, en su quehacer cotidiano, en el entramado que conforman a su andar (Ingold 2001).

En el caso específico de análisis desarrollado en el trabajo, el abordaje relacional es sustento conceptual a partir del cual se pueden describir los modos en que la gente transita dentro y fuera del chañaral, representadas a través de una red de flujos y contraflujos que relajan, tensionan y en definitiva hilan un entramado ambiental desbordado de historias locales. En tanto, la mirada no está puesta en atributos propios de seres vivos autocontenidos, sino en el carácter fluido del proceso vital en el que los límites son sustentados gracias al flujo de materiales que corren a través de ellos.

El concepto de cosa descripto por Ingold (1993) y

\footnotetext{
${ }^{3}$ Las salamancas definidas como reuniones fantasmales, diabólicas, mágicas; que son explicadas por el autor como espacios de constitución e impugnación de identidades y relaciones políticas en un contexto de re-emergencia huarpe en Cuyo (Escolar 2012).
} 
representado por él en la figura de un micelio (como una tela ramificante de líneas de crecimiento), es una imagen que sirve representa no tanto a los principios y los finales, sino más bien a los nodos intermedios por los cuales dichos hilos recorren el estar-siendo la vida, reflejando la importancia de hacer foco precisamente no en la forma determinada (y por tal determinante) sino en la intensidad en que esos hilos texturales se conjugan y relacionan, y con el ritmo en que lo hacen. El desafío es reemplazar esta concepción finalista del proceso de vida para representarla y ejemplificarla como una cosa, lo que en palabras del autor:

"Es en la esencia de la vida, el hecho de que no empiece aquí o termine allí, o conecte un punto de origen a un destino final, sino que se mantiene avanzando (keeps on going), encontrando un camino entre las múltiples cosas que forman, persisten y se rompen en sus trayectos. La vida, en resumen, es un movimiento de apertura, no de clausura." (Ingold 2012b : 35)

Lo que usualmente denominamos ambiente es en esencia esta trama de cosas. Gente que vive sus vidas en un mundo rodeado por otra gente, objetos y lugares, que a su vez están entretejidos por sus antecesores a través de actividades intencionales que antes configuraron las condiciones con las que luego se desarrollarán sus sucesores. Ingold desarrolla su pensamiento en el mismo sentido de Kusch (Kusch en Wright 2008) al plantear que siempre estamos instalados en alguna parte; no como seres sino como devenires, cada uno instanciado como una cierta forma de vida en el mundo, o mas bien como

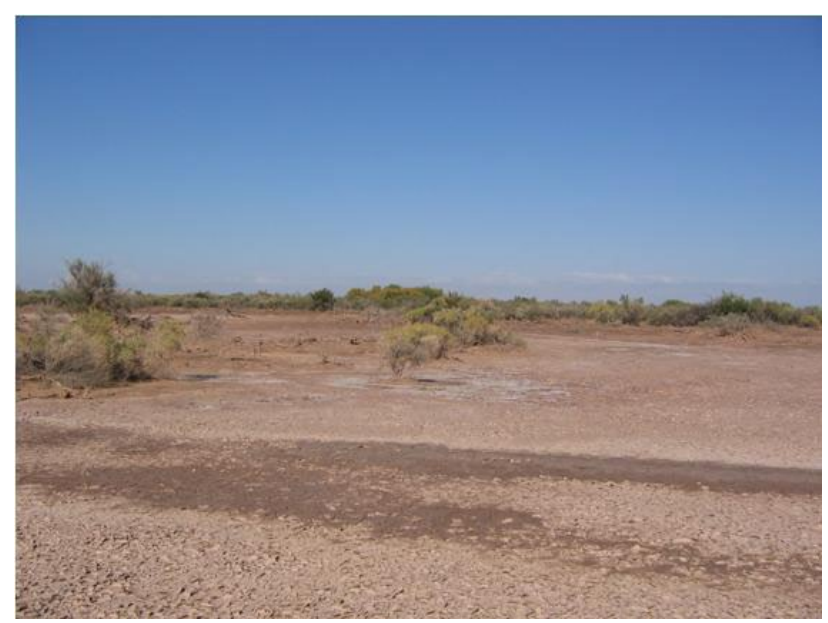

Figura 1: Una vista hacia el campo en la región de las lagunas de Guanacache. En el horizonte se observan bosques de chañar (Geoffroea decorticans) o chañarales en flor, Noreste de la provincia de Mendoza, Argentina (FOTO: L. Besio).

Figure 1: A view to the field in the region of Guanacache lagoons. On the horizon we can observe forests of Chañar (Geoffroea decorticans) or Chañarales in flower, Northeast of the province of Mendoza, Argentina (PHOTO: L. Besio). una cierta forma de estar vivo hacia el mundo.

\section{Guanacache es la urdimbre}

"El chañaral es energía, por eso toda casa en el campo tiene cerca creciendo un bosque de chañar." ${ }^{4}$

El lugar en el monte del que haremos letra en este trabajo pertenece a la región de las Lagunas de Guanacache (departamento de Lavalle, noreste de la provincia de Mendoza), también llamada área de secano o "desierto" por haber quedado fuera del reparto hídrico y ser de difícil acceso. Fotografía de un espacio de llanura que muestra elevaciones en forma de médanos y depresiones en forma de grandes concavidades en el terreno, espacios que hacen de contorno, y a su vez son contorneados por asentamientos humanos llamados puestos 5 .

"Laguneros", como también son llamados los pobladores que habitan la región, viven en un espacio que hasta las primeras décadas del siglo XX estaba conformado por un complejo sistema de lagunas que fue desecado mayoritariamente por la captación de aguas arriba durante el proceso de ampliación de la actividad vitivinícola en la provincia, intensificada hacia fines del siglo XIX y representada en la Ley de Aguas promulgada en dicho periodo. Es así, como el desecamiento de las lagunas se traduce en un caso de desigualdad geográfica local que a pesar de ser extensamente desarrollado por los ámbitos técnico científicos, de políticas públicas y literarias, ha sido escasamente abordado en su carácter político, social y cultural (Escolar y Saldi 2013; Saldi 2011).

Desde la década de 1990, los pobladores de la región pasaron a personificar un sujeto colectivo huarpe con reconocimiento de vínculos de continuidad biológica, cultural y social con poblaciones indígenas del pasado; dando comienzo a un proceso de emergencia de un pueblo huarpe legítimo, considerado extinto hasta el momento. Los pobladores autoadscriptos indígenas se transformaron en el principal referente del debate sobre la nueva vieja existencia de indígenas en la zona, al mismo tiempo que en un objeto de promoción y disputa por parte de diversas agencias y organizaciones (Escolar 2007, 2010). En tanto que la numerosa producción científica abocada al estudio sobre conocimientos y usos "tradicionales" en el marco de desarrollo de actividades sustentables en la región, ejemplifica un modelo de percepción ambiental sustentado con herramientas metodológicas y

\footnotetext{
${ }^{4}$ Relato contado en voz de AM sobre la apreciación enunciada por un poblador de la localidad de Asunción, en la región de Guanacache (Extraído de mi libreta de campo, Julio 2015).

5 La población en toda el área de Lagunas del Guanacache se encuentra agrupada en cuatro localidades (Asunción, San José, Lagunas del Rosario y San Miguel) con una concentración mínima de habitantes que no supera las 100 viviendas; mientras que el resto de la población se encuentra dispersa en asentamientos en torno a los denominados puestos, los cuales son unidades domésticas que tienen como principal economía el pastoreo y la venta de ganado menor, y en algunos casos, mayor.
} 
epistemológicas propias de occidente, sobre lo que podría dar en llamar el monumento geográfico de lo "huarpe" y lo "natural".

En los próximos párrafos iré armando la idea del presente escrito cual si fuese una urdiembre, matriz sobre la cual se van tejiendo texturas de una historia protagonizada por relatos de "tradición" de principios de siglo XX, y por estudiosos científicos y estudiosos "laguneros", todos salpicados por chañarales en distinto grado. Bajo el supuesto de que las prácticas y saberes trasmitidos que implican el uso de plantas podrían no estar refiriéndose sólo a elementos naturales formalmente ordenados por fuera del sentido social al que dan sentido y contexto, propondré desde una mirada etnográfica construir el carácter histórico y contextual que adquieren los chañarales. Mi foco de estudio son las plantas curativas y es mediante el empleo de teorías ontológicas híbridas de sociedad/naturaleza que intentaré explicar las formas relacionales en que los bosques chañarales estarían siendo convocados por la gente local como ámbitos de resistencia espiritual y material para la cura que sincronizan alianzas entre mundos pasados y a la vez presentes.

\section{El chañar: antecedentes de un individuo con tradiciones botánicas, ecológicas y culturales}

Estudiosos botánicos y etnobotánicos de la región han explicado según pautas de aplicación del modelo botánico occidental ${ }^{6}$ las virtudes del chañar y su homologación al correspondiente nombre local o "tradicional", incorporando los detalles ecológicos en que medran las especies junto con las observaciones culturales referidas al uso y conocimiento local indígena de dichas especies.

El pensamiento occidental botánico tradujo ya desde el año 1832 a chañar como la denominación local, vulgar o tradicional de una especie originalmente bautizada Gourliea decorticans por el Dr. John Gillies y luego reposicionada en la sistemática botánica por Arturo E. Burkart con el nombre de Geoffroea decorticans. A continuación detallaré en breve la historia curiosa respecto del nombre original $G$. decorticans como muestra de las pequeñas acepciones personales que atraviesan y se manifiestan en el seno de la clasificación taxonómica planteada por Carl von Linné (o Linneo) y continuada con rigurosidad científica por los botánicos sistemáticos que le sucedieron.

El libro Piedras de Afilar (Dawe 1988) narra la historia de vida de Dr. John Gillies, un médico escocés que viajó por razones de salud a la Argentina en 1820 y se estableció en Mendoza. Durante su estadía hizo excursiones botánicas y cuando regresó a Escocia se dedicó a estudiar y describir

\footnotetext{
${ }^{6}$ Tal es el caso de Adrián Ruiz Leal (1972), Fidel A. Roig (1993, 2001) y M. Cecilia Montani (2008, 2012; Montani et al. 2010), quienes han estudiado y descripto a las especies vegetales siguiendo la clasificación moderna construida sobre el sistema propuesto por Linneo: el sistema de identificación taxonómica.
}

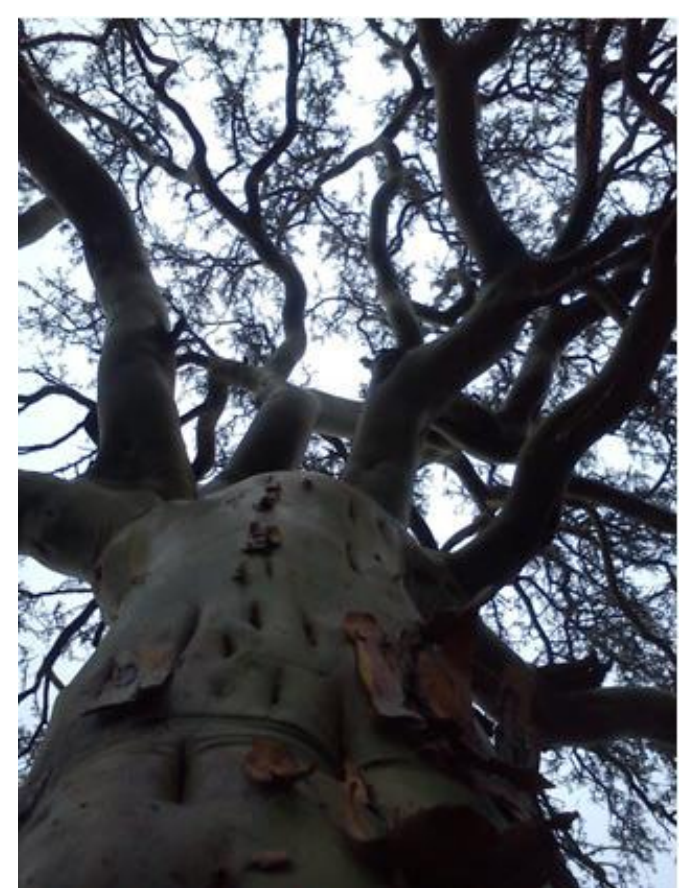

Figura 2: Un ejemplar típico de chañar (Geoffroea decorticans), región de lagunas de Guanacache al Noreste de la provincia de Mendoza, Argentina (FOTO: Nicolás M. Sanz)

Figure 2: A typical specimen of Chañar (Geoffroea decorticans), region of Guanacache lagoons to the Northeast of the province of Mendoza, Argentina (PHOTO: Nicolás M. Sanz).

junto a estudiosos botánicos las plantas que había recolectado en su viaje por Argentina. En Mendoza, Gillies comenzó un herbario para acomodar las plantas y semillas que había recolectado en su viaje desde la provincia de Buenos Aires, y en el que eventualmente incluyó los muchos especímenes reunidos en sus frecuentes excursiones por la cordillera de Los Andes y en la provincia de Mendoza.

Robert Gourlie (Glasgow, Escocia) es un joven botánico que con el tiempo deviene en su discípulo, de modo que cuando Gillies es obligado a viajar a Europa deja en sus manos todo su trabajo botánico para que Gourlie lo continúe en su ausencia. Mientras tanto en Escocia, Gillies toma contacto personal con un estudioso de la botánica sistemática: William Hooker, a quien pone a disposición sus colecciones de herbario para preparar las descripciones de aquellas plantas sudamericanas que podrían ser incluidas en ediciones futuras de la Miscelánea Botánica de Hooker. Es así como en el año 1832, en el volumen III de Miscelánea Botánica, se publica el trabajo Contribuciones a la flora de Sudamérica y las islas del Pacífico por W.J. Hooker y G.A.W. Arnott. Allí se describen cerca de cuatrocientas plantas de Sudamérica. El arbusto chañar es denominado por Gilles como Gourliea decorticans en honor a su amigo Robert Gourlie y haciendo referencia en su epíteto a la propiedad visible de descortezamiento de la planta. Triste de contar, Gourlie nunca se entera en vida de la dedicatoria porque había fallecido en Mendoza 
poco antes de la publicación de este trabajo.

Años más tarde, Jorge Hieronymus (1879), un botánico alemán que desempeñó gran parte de su carrera botánica en Argentina, dio a conocer a G. decorticans como elemento vegetal medicinal en un catálogo de las plantas de utilidad etnobotánica en Argentina titulado Plantae diaphoricae florae argentinae. Allí describe al público científico la flora de las regiones argentinas usando la lengua latina como era de costumbre en la disciplina botánica para las descripciones de nuevas especies. De esta forma, la especie vegetal aún con identidad $G$. decorticans entra renovada al campo científico, esta vez según sus cualidades fitoquímicas y terapéuticas.

El hilo que une y atraviesa temporalidades, autores, títulos y párrafos, naturalidades y sociabilidades en este ensayo, y que tiene como móvil ejemplificador al chañar, alcanza sus razones ecológicas de la mano de estudiosos botánicos de la región. Así, la especie vegetal es descripta conformando "isletas" en las ciénagas, donde en algunas partes origina matorrales extensos prácticamente impenetrables por el hombre y los animales; considerada en varias zonas de Argentina como una leñosa arbustiva invasora, principalmente en zonas semiáridas y subhúmedas; indicadora de salinidad y de napa freática poco profunda en zonas áridas. Por otro lado, los autores muestran a los bosques como propicios para la protección del suelo contra la erosión, siendo los montes de chañar aptos para la fijación de médanos y para la recuperación de suelos salinos. En tanto a los aprovechamientos populares,

"su madera firme es útil en la construcción de viviendas rústicas de quincha, cabos de herramientas y otros enseres domésticos y como combustible, pero su uso mayor, que es el que ha acarreado su intensa explotación, es el de postes y barretas para alambrados, para lo cual se sacrifican plantas muy jóvenes que tienen troncos de no más de 3-4 (5) cm de diámetro con lo que se produce una verdadera devastación" (Soria et al. 2007)

Sobre las razones curativas, autores mencionan la elaboración de arrope de sus frutos como el mejor remedio contra la tos, catarro bronquial, asma y que los niños en el campo aprecian mucho (Roig 2001; Montani 2008, 2010, 2012). La propiedad de tóxico embriagante al enunciar que sus frutos fermentados dan una aloja de virtudes curativas frente a disfunciones respiratorias (Roig 2001). El empleo del decoctado de corteza y hojas como emoliente y antiasmático (Ruiz Leal 1972); y de la infusión de flores de chañar junto con flores de "vira-vira" y "atamisque" para calmar los síntomas de gripes y resfríos (Montani 2012). La mención del chañar en la cura por el rastro de "hernias de ombligo" de niños recién nacidos. "... se marca con un cuchillo el rastro del pie o la mano de la persona sobre la corteza del chañar. (...) A medida que la herida del árbol vaya cicatrizando la hernia del niño se irá sanando" (Montani 2012 : 263). En relación al consumo de sus frutos como alimento de emergencia, la tradición es transcripta por Roig (2001) haciendo uso de Las memorias del excautivo Santiago Avendaño entre los años 1834-1874 (editadas en un libro cuyo autor es Meinrado Hux en 1999). Entonces le dijo Caniú:

"bueno sería que trates de juntar fruta de chañar para llevar contigo. Irás todos los días muy temprano a la isleta grande de chañares a cuidar para que nadie coseche de ella..." Y luego el cacique Pichuiñ, le recomienda..."que de la fruta que fuese secando juntase cuanto pudiera para llevarla en el viaje" (p.125).

Menciones de las razones medicinales asociadas al chañar que también están presentes en algunas de mis notas de campo, cuando le pregunto a Betina si los chañares ya estaban en flor, a lo que ella responde sin mayores especificaciones: "algunos sí... depende de dónde esté usted... porque por los médanos camino a mi casa hay algunos que ya están... algunos sí ya están en flor". Ambas somos beneficiarias de viajar en el asiento delantero de la camioneta que hace de transporte local que "te entra" o "te saca" del campo, en el momento mismo en que el monte se muestra reverdecido. Ante la imagen privilegiada, iniciamos una conversación entorno a su relación con las plantas y su conocimiento de ellas, para emplearlas como medicina para sus nietos que viven en un puesto alejado y quedan a su cuidado semana de por medio. Me cuenta...

..."para la tos té de flores de chañar y flores de atamisqui (dulces) con azúcar quemada. Usted las junta cuando hay y las seca en una bolsa de harina al sol, así las guarda para cuando necesite. Para la tos bronquial, para cuando a uno de mis nietos se le cierra el pecho y debe vérselas sin el paff, le preparo una infusión de la entrecáscara (me explica que debe extraerse de la madera externa del palo, y con un cuchillo se va raspando el palo y eso es lo que se cuece). Por ejemplo, del chañar no se usa ni la cáscara que se desprende ni la madera verde, sino la entrecáscara que es lo hay adentro que hay que raspar con el cuchillo. Se llama té de siete palos: ramas de retamo, atamisqui, jarilla, lámaro (aclara no de algarrobo dulce), chilca, chañar y palo azul, a lo que le agrega azúcar quemada y deja reposar; al día siguiente se calientan tres cucharas de arena al fuego de la hornalla y en una tela se la pone en el pecho del niño acostado, y se le da el té de siete palos y ahí se lo tapa y que descase."

(Extraído de mi cuaderno de campo, Septiembre 2014).

De las flores que conviene recolectar, Betina aclara que las mejores flores de chañar no son las cuaresmeras sino las de noviembre o diciembre, porque tanto el algarrobo cuaresmero como el chañar cuaresmero son débiles, y 
ya no son tan dulces. Cuando pareciera entender que mi interlocutora está hablando de plantas que por su fenotipo adaptado a ciertas condiciones ambientales son a veces cuaresmeros y a veces no, me encuentro con el relato de Eduviges y Petra quienes alguna vez me había comentado que para hacer el arrope de chañar cuaresmero el mejor fruto es el primero, también el de la primer floración "porque tiene más fuerza, y es más dulce y grande". En aquel momento, ellas me explicaban que los chañares a veces son cuaresmeros y a veces no, depende del año y de las lluvias, y de cierta situación o contexto inmediato explicado por ellos con cierta magia: "el chañar cuaresmero es el mismo que el chañar no cuaresmero. Se llama chañar cuaresmero cuando "prende", cuando no sólo da fruto en primavera sino que después de las Iluvias de verano vuelve a dar fruto a fines de verano... en febrero". (Extraído de mi cuaderno de campo, Mayo 2014).

\section{"Ya soy vieja para creer a lo moderno": historias de cura, magia y ritual}

“El colonialismo impuso sus propias categorías de tiempo sobre los pueblos y espacios conquistados. Éstos carecieron de toda oportunidad para hacer públicas sus interpretaciones históricas, su propia definición de "historia". Por supuesto que contaban con ellas, pero sus textos eran heterodoxos (orales, actuados, recordados, soñados); su alcance se resumía a lo local,

a veces a lo regional; éstas expresaban modos de ser-en-el-mundo ajenos a la percepción europea de la duración"

(Kusch en Wright $2008: 41$ )

En la zona de los Altos del Retamo, nos pasamos del camino de entrada para llegar al puesto de Juan porque perdimos de vista la marca: una casilla de una virgen en orientación Este mirando hacia el pueblo Lagunas del Rosario, así que de arriba del vehículo debemos recurrir al GPS. Esta vez, el puesto no tiene ninguna señal que lo identifique en la distancia ante nuestros ojos plagados de urbe e inmersos además en la dificultad de atravesar una zona de profundas hondanadas y altos médanos,

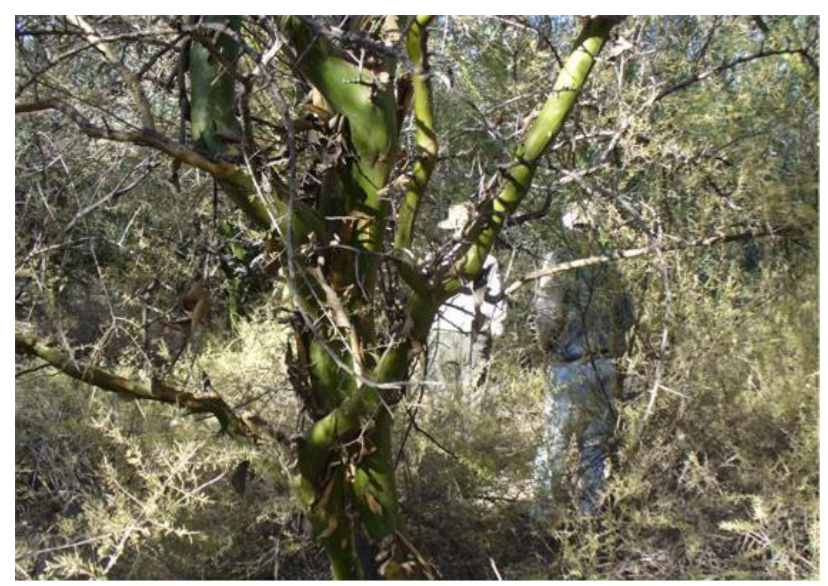

elevaciones que además existen movedizas siguiendo remolinos de viento propios de la zona.

“Llegamos pasadas las 12 del medio día. Pasamos y nos sentamos junto a su mesa.iQué cantidad de moscas! Yo nunca había visitado el campo en esta época, no conocía tal cantidad de mosquerío junto. Juan nos aclara que son las moscas del corral. Si me sentaba junto a la ventana donde daba el rayo de sol, más moscas. Había que elegir entre estar sentada a la sombra y sufrir la fresca que mantienen el adobe, o abrigarse con el solcito pero en compañía de las moscas que casi no te dejaban abrir la boca porque se volvían tu bocado. Elegí la sombra. Todo sobre la mesa bien tapado, bolsitas, frascos de malta con yerba y azúcar, todo al resguardo de ellas...las moscas. Juan cuerpo resuelto, en el lado de la mesa en que parece acostumbra sentarse, enfrentado a la puerta. Nosotros en dos sillas del lado opuesto de él, a espaldas de la puerta. De tal modo, que cada vez que entraba el burro a comer de la bolsa de trigo o de la de cáscara del cereal, mi curiosidad me obligaba a darme vuelta en respuesta al sonido que el animal hacía en su entrada. En cada oportunidad, Juan se levantaba y lo abrazaba de la cabeza con mucha suavidad y lo sacaba afuera. La escena se dio repetidas veces, asumiendo yo que la visita de los animales al interior de la casa y el posterior encuentro con Juan mostrándoles el camino a afuera, era parte de la cotidianidad."

(Extraído de mi cuaderno de campo, Lagunas de Guanacache. Mayo 2013)

Miradas que se cruzan y comienzan a reconocerse entre silencios. Juan reafirma su ausencia de conocimiento sobre plantas medicinales. Y sí menciona el saber que sus ancestros tenían sobre plantas: "en tiempos pasados...mi papá, mi mamá, mi tío", o directamente generaliza "...los viejos de antes" o "los huarpes". Entrelineas de un relato en tiempo pasado que entremezcla el presente, menciona un hongo que sale de la tierra como si fuera una flor, dice: "el del polvito naranja es el mejor, con el que se hace una pasta y se usa para cicatrizar, si uno se lo pone al animal en la herida, rápidamente cicatriza y hasta no deja marca de la herida". Luego cuenta que "los viejos que sabían de usar las plantas deben haber sabido de probar no más, y así se iban rescatando usos y formas de curar". Cuando narra sobre las plantas y los usos que él conoce se lamenta de no haber aprendido más sobre los conocimientos que tenía su madre sobre plantas, porque ella sabía mucho y hacia curaciones con las plantas; introduciendo en la

Figura 3: Relatos de cura y prosperidad bajo la cubierta de un chañaral. Localidad de Lagunas del Rosario, región de Guanacache, Noreste de la provincia de Mendoza, Argentina (FOTO: L. Besio).

Figure 3: Stories of healing and prosperity under the cover of a chañaral. Locality of Lagunas del Rosario, Guanacache region, Northeast of the province of Mendoza, Argentina (PHOTO: L. Besio). 
conversación el término condimentos curativos. Según sus explicaciones, dichos condimentos curativos eran preparados con plantas, hongos y se refieren también a la cura de palabra, y estaban reservados para la cura de animales y de plantas.

La conversación con Juan se mueve lentamente del pasado de sus ancestros a su presente, hilvanando temas desde lo medicinal a lo curativo, y de lo humano a lo no humano.

"La huáchara es remedio para la anemia; el retortuño es otro remedio para la anemia y además la agüita de sus frutos da energía; la pinchagua que son los arbustitos espinosos de frutitos anaranjados...rojizos, amarillos y negros; la zampa blanca, zampa crespa y zampa verde son buenas para los animales; la verde no la comen mucho porque es más amarga y la mejor es la crespa, además la crespa está muy frondosa cuando los otros forrajes están secos, en invierno. La vidriera es otra planta que enverdece su follaje en invierno. (...) la penca "una planta misteriosa que usaban los indios, son como víboras entre las plantas". Cuenta que se ocultan y mimetizan como víboras verdes con escamas amarillas, "uno las ve de lejos por sus flores amarillas, después se buscan y ya no están más". Y además detalla "tienen el mismo olor que el aloe vera, sus flores son dulces... a veces le saco un pedacito de pétalo a la flor y lo como".

(Extraído de mi cuaderno de campo, Lagunas del Rosario. Mayo 2013)

Salimos de la casa y nos adentramos al campo. Lentamente aunque sin rodeos, nuestro anfitrión nos introdujo a una interpretación ecológica profunda del campo y del contexto real de vida que gira entorno de trayectorias de la gente que tienen que ver con la convivencia del uso y manejo de los recursos forrajeros por ellos llevados a la práctica. Sobre el campo y sobre las políticas estatales implementadas en dicho ambiente en la región. Allí Juan daba su perspectiva sobre las plantas forrajeras que para él podrían ser buenas para emplear en el proyecto de reforestación del monte?

"la zampa y la pichanagua, porque crecen fácil y sin problemas, a los animales les gustan mucho, y tienen un ciclo de vida de siete años, o sea, que recién al sexto año comienzan a palarse y a perder hojas para luego morir... Además, son de simple manejo porque ambas tienen la característica de no dejar que otras plantas crezcan cerca. La zampa, sé donde podríamos ir a recolectar semillas a fines de temporada." (Extraído de mi cuaderno de campo, mayo 2013).

\footnotetext{
${ }_{7}$ Proyecto otorgado a la Comunidad Huarpe Lagunas del Rosario en el año 2013 en el marco de la Ley de Presupuestos Mínimos para la Protección de los Bosques Nativos, con intenciones de disminuir procesos de deforestación del monte en lagunas.
}

El pasaje de aquel atardecer en las conversaciones del campo nos encuentra en los contornos de un potrero devenido hoy en chañaral. Juan nos invita a transitar el espacio, tarea que obligaba revertir la verticalidad en nuestros cuerpos e ingresar agachados avanzando "en cuatro patas". La memoria escrita de aquella tarde relata:

“Estábamos en un espacio de bosque que antes había sido un potrero. Cuesta entrar. Ninguno de los chañarales antes visitados por mi presentó tanta densidad de arbustos espinosos como esta comunidad. Tanta espina que cuesta penetrarlo, agachados y en cuatro patas esquivando plantas de lámaro y piquillín del campo. Pudimos hacernos un huequito para conversar bajo su dosel... Juan nos cuenta de las ceremonias que se realizaban en los chañarales: para la prosperidad de los cultivos velaban toda la noche con rezos, haciendo un dibujo en la tierra de la huerta en escala pequeña, incluso a veces se ponían semillas sobre el dibujo en los sitios en los que iría en la huerta esa planta, se las regaba, y allí se quedaba la gente velando y rezando toda la noche. Se acuerda Juan que hubo veces que salieron plantas de las semillas que quedaban en la tierra luego de ese ritual. Entonces uno se encontraba con plantas de melón dentro de los chañarales, y de otras plantas también. Allí nos comenta de las diferencias de productividad de los huertos que manejaban sus tíos, abuelos y su papá, en los que él recuerda de niño haber puesto tres semillas por hoyo y salían las tres, en cambio, en los huertos para los que trabaja él ahora, cuenta "se ponen cinco semillas por hoyo y a veces no sale nada y a veces salen las cinco... entonces allí uno tiene que estar separando las raíces de las plantas con cuidado para que no se mueran". En cambio su abuelo sabía muy bien distinguir las semillas buenas de las semillas malas. Con sólo mirarlas sabía si dentro había aire o estaba la semilla, entonces el año anterior, separaban una cantidad de semillas todas prósperas y sembraban sólo esas, sabiendo que saldrían todas. (...) Otra ceremonia que cuenta Juan que tiene lugar en la zona de lagunas de Guanacache es la cura de cultivos por medio de la palabra, "entonces los gusanos del huerto se quedaban muertos en la planta... eso sí, después que se hacia la cura de palabra, no se las podía mirar a las plantas, recién al día siguiente se veían los gusanos muertos secos". Además Juan cuenta que se hacia la cura de palabra a cualquier planta del campo, con la energía de la palabra del curandero mientras salpicaba la planta con una ramita mojada."

(Extraído de mi cuaderno de campo, Mayo 2013)

En la corporalidad de sus palabras y en la textualidad de su relato, Juan no distingue ni siquiera en lo aparente al humano por un lado y el entorno ambiental no humano por el otro, sino que entabla una relación entre seres que dialogan dentro de un sistema amplio de cura: la cura de 
personas a animales y a otras personas a través de las plantas y a través de la energía en la palabra del curandero, pero también la cura de plantas del huerto o del monte, por medio de la palabra del curandero con el uso de una rama extraída del monte y mojada con agua.

Formas de cura que colmaban el aire de cierta magia, y que se volvían aire en movimiento cuando la luz del sol agotaba su llegada y daba comienzo el atardecer. Vivencias y percepciones que daban lugar y sentido a la emergencia de un sin razón de contundencia simbólica que apuraba nuestra retirada de aquel espacio de chañaral. Momentos ambiguos en que la apariencia cenicienta de los palos de las plantas y sus hojas tomaban volumen sin contrastar con el color del suelo, mareando el paso. La necesidad de un retorno a mi mundo ontológico en el que el suelo es cubierta que distancia la tierra del cielo, y disgrega lo real de lo fantástico en términos de causalidad. Asumiendo la imposibilidad de asumir una pronta salida del entorno del chañaral, optamos por adentrarnos en la propuesta interpretativa de Ingold (1993, 2001), intentando transitar aquel atardecer en el chañaral como si fuera un acto constante de "improvisación" en el que emergen acciones que en-habilitan la posible existencia de percepciones que abren la escucha a un relato que está-siendo y abandonan toda relevancia de incorporar seres en clausura.

La idea de asumir a la percepción como herramienta fundamental de conocimiento es planteada en un estudio de Surallés (2004), quien en sus propios términos explica:

“Una persona adherida al mundo, una persona que percibe y actúa. ... esta implicación de la persona sensible en el mundo supone que está sometida a estados cambiantes, reflejo de los estímulos que recibe de la realidad, al mismo tiempo que indisociablemente disuelta en su entorno (p. 140)".

En tanto, el autor propone interpretar el recorrido diario de las personas en su contexto inmediato de vida, como si el espacio fuera un sistema de orientación que permite explorar un medio en dinámica constante, ofreciendo al caminante "... los elementos que le permiten desencadenar la dialéctica entre la percepción y la acción necesarias para avanzar en este medio ambiente (p. 156)".

Un diálogo de eventos mágicos bajo las sombras de los chañarales que enuncia vivencias transmitidas en tiempos pasados y percibidas en nuestros cuerpos un tiempo actual; eventos mágicos que aparecen escurriéndose de cualquier clausura a la realidad que representan. Chañarales que constituyen a la vez que destituyen lo concreto y lo real de su mundo.

En el mismo tono de inscripciones sobre magia y ritual, propongo la lectura de la siguiente historia narrada por una mujer campesina a una maestra en una localidad rural del este de la provincia, quien a su vez la transcribe y decide titularla "temor a las brujas". (DE.: Doña Edelinda;

\section{M.: Maestra) ${ }^{8}$.}

“M.:- ¿Por qué no mandó su niña a la escuela doña Edelinda?

DE.:- No se pudo señora, tenemos los niños enfermos de yapa asustadas, porque anoche pasaron las brujas (a) las risotadas.

M.:- ¿Pero cómo es eso, cree en las brujas?

DE.:- Si señora como no he (de) creer, más ahora que está la Rosa para casarse, nos andan persiguiendo, de seguro alguna que tiene interés en el turco.

M.:- Pero esa no es una razón para creer en brujas.

DE.:- Yo creo porque en tiempo de mis abuelitos, que Dios los tenga en la gloria, nos pasó una mano muy seria con las brujas.

M.:- Cuénteme...

Muy chicos quedamos a cargo de nuestros abuelos, por el fallecimiento de nuestros padres, estos eran campos montañosos, puros chañarales, teníamos unas cuantas lecheras, los muchachos traían todas las tardes los terneros a encerrar, por la mañana buscaban las vacas que siempre dormían en la primera isleta de chañares. Esa mañana hicieron fuego y enseguida fueron a traer las lecheras. Mientras las juntaban encontraron un pájaro negro grande de los que se llaman jotes durmiendo junto a un árbol. Lo tomaron, jugaron con él, por fin lo llevaron a las casas a la cocina. Allí siguió la diversión arrimándolo al fuego, uno le quemaba las alas, otro, le enterraba el pico en la ceniza, aquel le rescoldaba las patas, hubieran seguido en sus crueldades si no se levanta mi abuelito. Quien les hizo que lo fueran a dejar donde estaba, él ya comprendió que era una bruja que le había tomado el día sin llegar a su casa.

Al otro día, muy temprano, estaba cebando mate, mi abuelita haciendo tortillas, cuando dijeron en el patio -¡Dios gracias!- ¿Qué se les ofrece? -No está don..... (mi abuelito).-Sí- Dígale que dice doña Eufrasia que vaya para que vea en qué estado que la han puesto sus nietos-. Los que venían eran dos niños, que criaba la vecina esa.

Fue mi abuelito y la halló en cama, toda quemada la cara brazos y piernas, entones le confesó que ella era el pájaro que encontraron lo niños, que fue muy lejos esa noche, que la tomó el día si alcanzar a llegar a su casa, y por lo tanto sin poder volverse persona.

Al otro día murió, sin alcanzarse a confesar. Ya ve si tengo razón en creer en las brujas. Y hasta ahora las hay. Dice don Palorma, que el otro día iba él de a caballo, cuando se le acercó un pájaro negro y le tiró de las mechas.

M.:- Sí, ¿tienen mucho poder las brujas?

DE.:- Bien grande, necesitan muchos años de estudio y conseguir juntar los sesos de siete animales de los más venenosos conocidos.

M.:- Bueno muy bien, mándeme los niños a la escuela y

${ }^{8}$ ENF. Buenos Aires, 1937. Relato de doña Edelinda de 40 años, inscripto
por la maestra Felisa R. de Oro en la localidad de Campamentos (Rivadavia, Mendoza). 
no crea en brujas.

DE.:- Ya soy vieja para creer a lo moderno. No voy a hacer como mi hija María, que cocina por los figurines (recetas de cocina que vienen en las revistas) para no parecer antigua.

Entre los momentos en que se hace letra y sonido la transcripción del relato de doña Edelinda en el que asocia creencias de brujería con los chañarales, se entremezclan conversaciones con Doña Rosa y Aníbal que emergen aisladas en tiempo y espacio pero que encuentran profundidad argumentativa en el presente estudio al hilar junto a Juan y a Doña Edelinda sobre el sentido social espiritual otorgado a los bosques de chañar.

"Andábamos con intenciones de visitar aquel chañaral recomendado por Doña Rosa sobre tierras removidas de arenas, polvo y blancura propia del salitre y del tenue verde de jume, vinagrillo y vidrier ${ }^{9}$, para repentinamente volvernos a nuestros pies y darnos cuenta que ahora caminábamos sobre bloques duros de un amplio rompecabezas arcilloso indicativo de viejos-nuevos cauces de agua que por allí decantan y atraviesan el campo camino a las lagunas. Travesía que es parte del cauce del río Tulumaya, sinuosas correntadas conviviendo con las ya renombradas "isletas de chañares", espacios que emergen del terreno como cuentas o perlas que dan figura al hilo de agua que por debajo, y sólo a veces superficialmente, se ve. A lo lejos en la amplia llanura, las nubes de catas como el emblema que nos permitió llegar de regreso a la casa de Doña Rosa. A nuestra llegada a la casa luego de un recorrido por el campo aledaño, nos esperaban los mates que acompañan la fresca del atardecer y el cierre del día que pasó. Entre idas y venidas de mates, la anfitriona del encuentro rompe su silencio y nos pregunta sobre las plantas que habíamos visto en el camino por ella recomendado. Ante cada descripción nuestra sobre las plantas encontradas, Doña Rosa no pronuncia asombro, más bien al contrario, nos explica con soltura la permanente presencia de esas plantas en aquellos sitios y junto a qué otras plantas suelen encontrarse, y en las formas que adoptan en distintos periodos de su vida y según el estado general del ambiente. Ella es una mujer mayor que vive sus días con una enfermedad crónica que le permite desplazarse físicamente escasos metros en torno a la casa. Por tanto, veo en aquel día una memoria atenta a reconstruir continuamente su imagen del campo, curiosa de nuestras percepciones; cual si cerrara los ojos y se permitiera caminar junto a nosotros aquellos senderos polvorientos marcados por la huella cotidiana de las cabras" (Extraído de mi cuaderno de campo, abril 2014)

\footnotetext{
${ }^{9}$ Formas locales de llamar las plantas que refieren a los nombres científicos: Allenrolfea vaginata (jume), Salicornia ambigua (vinagriIlo), y la especie Suaeda patagonica (vidriera)
}

En la misma locación geográfica cuyo ícono indica el chañaral aquella vez recomendado por Doña Rosa; hilamos con la palabra y la guía de Aníbal, hijo de Doña Rosa, conocedor y sobre todo entusiasta en retratar para nosotros andanzas propias del campo, y sus pormenores cotidianos. Mis notas retratan de la siguiente manera el momento:

“Bajo el chañaral, Aníbal cuenta que a ese paro lo llaman la cocina, a simple vista denotado por un espacio descubierto y desprovisto de cualquier vegetación y con unas rocas dispuestas conformando un asiento frente al lugar de lo que Aníbal nos traduce como antiguo sitio de fuego. Sobre la imagen de mi sorpresa, Aníbal responde diciendo que allá "cocinaba un señor que andaba por el monte y que vivía de lo que el monte le daba". Cada tanto el señor hacía trabajos para su papá y es precisamente por esto último que su familia sabía de su presencia en aquel chañaral. Continúa Aníbal y nos cuenta que por toda el área de las lagunas existen hombres que viven en el monte y del monte, al igual que este señor. Momentos más tarde, cuando yo aun intentaba representar la idea en mi mente de la cocina dentro de aquel chañaral, Aníbal nos confiesa que es también en los chañarales en que acontece el momento de iniciación de los hombres, que consiste en dejar solo al joven que se inicia, en una noche de viento y bajo un chañaral. A lo que agrega "...la corteza del chañar cuando hay viento, suena de formas que estremecen"

(Extraído de mi cuaderno de campo, Noviembre 2012).

Una posible forma de incorporar en el análisis los elementos que permiten el dialogo entre Juan, Doña Rosa, Aníbal, Doña Edelinda y el ambiente por ellos transitado es señalar "marcas que podrían ser entidades o eventos que después de aparecer ante los humanos modifican su campo existencial" (Wright 2008 : 39). Considerando que la emergencia de sentido de dichas marcas está necesariamente ligada al mundo social del intérprete, al suelo sobre el que emergen y dentro del contexto relacional en el que circunstancialmente son contruídas. Podemos pensar en la magia como una marca que remite:

"... al momento mismo de realización de fenómenos, sujetos sociales y políticos en contextos en que tales fenómenos son aún epistemológica y ontológicamente inaprensibles, y donde una porción, combinación o posibilidad de lo existente pasa a constituirse como real. La magia asociada de este modo a resistencia de las fuentes de saber. Como espacio y tiempo de producción de nociones de lo real por constitución y destitución de identidades y sujetos políticos indígenas (Escolar 2012: 18-19)".

El chañaral y los eventos de magia allí dentro relatados, percibidos, y en tanto interactuando en la propia historia, 
comienzan a mostrarse como una cosa entretejida de brujería, cura y vínculos entorno al manejo comunitario del ambiente que trascienden el tiempo pasado y se enclavan en el presente.

Decantando la idea, la magia aparece dentro del chañaral abriendo el foco hacia una posible perspectiva ontológica que no puede ser explicada en términos de lo "natural" y lo "social" como esferas ajenas y separadas una de otra. El carácter vivo está así determinado por el principio relacional del ser que permite interpretar a Gourlie decorticans, Geoffroea decorticans, al chañar patero y al chañar cuaresmero despojados de la clausura que otorgan las líneas de su morfología botánica y adentrados en un mundo enmarañado por cosas, donde varios aconteceres se entrelazan y tejen una historia, una concepción de saber para la cura, y una realidad que los hace a ellos partícipes; tomando como premisa la imagen de las cosas escurriendo y desbordando siempre las superficies que las forman temporalmente en torno a ellas (Ingold 1993).

\section{Puntadas finales y a modo de conclusiones}

Entre líneas aparece el relato que da cuerpo al epígrafe general del trabajo y que declara un modo de cura que nos transporta sutilmente desde lo que podría traducirse como una receta casera con especificidad material en el uso de entidades biológicas con virtud medicinal, hacia un espacio entretejido de materialidad y simbolismo mágico desembriagante protagonizado en este caso por plantas y sesos de lechuzo...en pequeña cantidad. El propio escenario académico configurado con anteriores retratos de plantas medicinales vertiginosamente deviene en un universo difuso de representaciones de cura simbólica.

La imagen de un jote a orillas de un chañaral que al adentrarse el amanecer debió permanecer allí porque lo tomó el día sin poder llegar a su casa. Los muchachos hermanos de doña Edelinda lo encuentran y se divierten con él arrimándolo al fuego, hasta llegada la advertencia de su abuelo quien los obliga a retornar al malherido jote al chañaral. La noticia de que al día siguiente Doña Eufrasia muere por profundas quemaduras en todo su cuerpo sin alcanzar a confesarse. $Y$ el temor a las brujas que resurge y permanece en la vida de doña Edelinda, en parte porque ella ya es vieja para creer a lo moderno.

El temor a las brujas asociado a espacios de bosques me lleva a volver sobre propias notas etnográficas tomadas en diferentes instancias de campo. La curiosa intromisión de la cocina, espacio que cobija la historia de "un señor que vivía en el monte y de lo que el monte le daba", espacio de cocción de una realidad cotidiana que es recitada por Aníbal dentro y bajo el dosel del chañaral que protagoniza de habitáculo. Entidades boscosas que además articulan en momentos espirituales de iniciación de jóvenes en el campo, porque "la corteza del chañar cuando hay viento, suena de formas que estremecen". Pliegues más tarde aparece Juan y sincroniza pasado y presente asociados a prácticas rituales de cura y prosperidad de cultivos relatadas bajo la omnipresente sombra de los chañares.

Saliendo de momentos en el monte, y continuando la rutina del matecito tibio que acompaña el atardecer en el campo, reflexiono y me vuelvo consciente de que ningún recitado de andanzas prevista de eventos rituales me fue convocado por fuera de la entidad de los chañarales.

La rigidez contundente del suelo donde figuran formas y sombras, los descascarados troncos, sus espinas y dulces frutos renombrados por su carácter de tóxico embriagante con propiedades curativas; rincones todos presenciados por chañares; cual si fueran espacios tejidos de sociabilidad que están vivos en tanto habilitan prácticas rituales para la prosperidad de los cultivos, la iniciación de jóvenes, y la cura de plantas con otras plantas mediante la palabra del curandero habilitado para ello. O al contrario, espacios en los que es convocada la enemistad, la disputa y la muerte. $Y$ es en tanto, que podríamos interpretar al chañaral como un espacio de resistencia epistemológica del mundo, espacio de diálogo entre seres aliados tanto del mundo de los vivos como del mundo de los muertos que son convocados a hilar a su sombra y en la salvedad del pasado.

Mendoza, 30 de noviembre 2016

\section{Bibliografía citada}

Dawe, O. 1988. Piedras de Afilar. The unfulfilled dream of a 19th-century scottish botanist in South America, Charles Skilton Ltd., Scotland.

Descola, P. 2001. Construyendo naturalezas. Ecología Simbólica y práctica social. Descola Philippe y Pálsson Gísli (coord.), Naturaleza y Sociedad. Perspectivas antropológicas. Primera parte, 101-123, Siglo XXI Eds. México, DF.

Descola, P. 2012. Más allá de la Naturaleza y la Cultura. Amorrotou Editores. Buenos Aires.

Descola, P., G. Palsson. 2001. Introducción. Descola Philippe y Pálsson Gísli (coord.), Naturaleza y Sociedad. Perspectivas antropológica. 11-36, Siglo XXI Ed. México, DF.

Escolar, D. 2007. Los dones étnicos de la Nación. Identidades huarpe y modos de producción de soberanía en Argentina. Prometeo Ed. Bs. As. Argentina.

Escolar, D. 2010. Acompañando al pueblo huarpe: luchas de representación y control político en la institucionalización de las Comunidades Huarpe de Guanacache, Mendoza. Gastón Gordillo y Silvia Hirsch Eds., Movilizaciones Indígenas e identidades en disputa en la Argentina, capítulo 7, 173-205, La Crujía, Bs.As. Argentina.

Escolar, D. 2012. El vórtice soberano: salamancas, políticas de lo extraordinario y la emergencia de los huarpes en Cuyo, Argentina. Nuevo Mundo Mundos Nuevos, Cuestiones del tiempo 
presente, (no. 12)http//nuevomundo.revues.org/64570. Última consulta: 22/04/2013.

Escolar, D., L. Saldi. 2013. Canales fantasma en el "desierto huarpe". Riego legal, discursos ecológicos y apropiación del agua en Cuyo. Argentina. Siglos XIX-XX. Rev. Agenda Social 7 (1): 68-94.

Encuesta Nacional Folclórica (ENF). 1937. Formalizada por la Facultad de Filosofía y Letras de la Universidad de Buenos Aires, Instituto de Literatura Argentina bajo la dirección de Ricardo Rojas en el año 1921. La edición de la ENF se completó en Buenos Aires, Imprenta de la Universidad (1937), donada por el Consejo Nacional de Educación al Instituto Nacional de Antropología y Pensamiento Latinoamericano (INAPL).

Hieronymus, J. 1879. Plantae diaphoricae florae argentinae, Boletín de la Academia Nacional de Ciencias de Córdoba 4 (3-4).

Hviding, E. 2001. Naturaleza, Cultura, Magia, Ciencia. Sobre los metalenguajes de comparación en la ecología cultural. Descola Philippe y Pálsson Gísli (coord.), Naturaleza y Sociedad. Perspectivas antropológicas. Segunda parte, 192-213, Siglo XXI Eds. México, DF.

Kalinsky, B., M. Carrasco, W. Arrue y G. Witteman. 1994. Si Dios es el mismo siempre. Paisajes, contrastes y pluralismo cultural en el ámbito de la salud. Sociedad y Religión 12: 79-96.

Ingold, T. 1993. The Temporality of the Landscape. World Archaeology 25(2): 152-174.

Ingold, T. 2001. El forrajeo óptimo y el hombre económico. Descola Philippe y Pálsson Gísli (coord.), Naturaleza y Sociedad. Perspectivas antropológicas. Primera parte, 37-59, Siglo XXI Eds. México, DF.

Ingold, T.. 2004. Beyond biology and culture. The meaning of evolution in a relational world. Social Anthropology. 12 (2): 209-221.

Ingold, T. 2006. Rethinking the animate, re-animating thought. Ethnos, 71 (1): 9-20.

Ingold, T. 2012a. Trazendo as coisas de volta à vida: Emaranhados criativos num mundo de materiais. Horizontes Antropológicos, 37: $25-44$.

Ingold, T. 2012b. Contra la Cultura, abrazando la vida: antropología más allá de la humanidad. Tim Ingold. Ambientes para la vida. Coversaciones sobre humanidad, conocimiento $e$ antropología, 35-52, Trilce Eds, Montevideo.

Kusch, R. 2012. El pensamiento indígena y popular en América y la negación del pensamiento popular. 113 pp., Editorial Fundación A. Ross, Rosario, Argentina.

Latour, B. 2007. Nunca Fuimos modernos. Ensayos de antropología simétrica. 224 p., Siglo XXI Eds., Bs.As.
Marconetto, M. B. 2008. Linnaeus en el Ambato. Límites del uso de la clasificación taxonómica en Paleoetnobotánica. Sonia Archila, Marco Giovannetti y Verónica Lema (Eds.) Arqueobotánica y teoría arqueológica. Discusiones desde Suramérica. Primera parte, 143-166, Ediciones Uniandes, Bogotá.

Montani, M. C. 2008. "Aporte al conocimiento etnobotánico en comunidades originarias: Sistematización del uso medicinal de plantas en la Comunidad Huarpe de Lagunas del Rosario, Lavalle (Mza., Arg.)". Facultad de Ciencias Exactas, Físicas y Naturales, Universidad Nacional de Sa Juan, Argentina, 112pp.

Montani, M. C. 2010. Raices Huarpes: Uso medicinal de plantas en la comunidad de Lagunas del Rosario, Mendoza, Argentina. María Cecilia Montani y Cecilia Vega Riveros (Eds.) 96 pp., Editorial Fundación Universidad Nacional de San Juan., San Juan.

Montani, M. C. 2012. Uso medicinal de plantas entre descendientes huarpes en la comunidad de Lagunas del Rosario (Mendoza, Argentina). Pastor Arenas, Etnobotánica en zonas áridas y semiáridas del Cono Sur de Suramérica. 251-270, Edición del CEFYBO-CONICET, Bs.As.

Palsson, G. 2001. Relaciones humano-ambientales. Orientalismo, paternalismo y comunalismo. Descola Philippe y Pálsson Gísli (coord.), Naturaleza y Sociedad. Perspectivas antropológicas. Primera parte, 80-100, Siglo XXI Eds. Bs. As.

Rival, L. 2004. El crecimiento de las familias y los árboles: la percepción del bosque de los Huaorani. Surallés A. y García Hierro P. (Eds.) Tierra Adentro: territorio indígena y percepción del entorno. Parte dos, 97-119, Grupo internacional de trabajos sobre asuntos indígenas. Lima, Perú.

Roig, F.A. 2001. Flora Medicinal Mendocina. Las plantas medicinales y aromáticas de la provincia de Mendoza, Argentina. 305 pp., Editorial EDIUNC, Mendoza.

Ruiz Leal, A. 1972. Flora popular mendocina. Deserta III. 296 pp., Editorial CONICET-IADIZA, UNCuyo, Gobierno de Mendoza.

Saldi, L. 2011. "Procesos identitarios, naturaleza y políticas estatales en el noreste de Mendoza (Argentina)". Facultad de Ciencias Políticas y Sociales, Universidad Nacional de Cuyo (FCPySUNCuyo) Argentina, 363 pp., FCPyS-UNCuyo.

Soria N. D.; Salomón M. A.; Rubio C.; y Fernández R. 2007. Herbario digital del secano de Lavalle. Laboratorio de Desertificación y Ordenamiento Territorial. LADyOT - IADIZA CRICYT. Mendoza.

Surallés, A. 2004. Horizontes de intimidad. Persona, percepción y espacio en los Candoshi. Surallés A. y García Hierro P. (Eds.) Tierra Adentro: territorio indígena y percepción del entorno. Parte dos, 137-162, Grupo internacional de trabajos sobre asuntos indígenas. Lima, Perú.

Wright, P. 2008. Ser-en-el-sueño: Crónicas de historia y vida toba. 270 pp., Editorial Biblos, Bs. As. 